New Bioink

\title{
A NEW BIOINK FOR IMPROVED 3D BIOPRINTING OF BONE-LIKE CONSTRUCTS
}

Adam C. Marsh ${ }^{1}$, Ehsanul Hoque Apu ${ }^{2,3}$, Marcus Bunn ${ }^{2,3}$, Christopher H. Contag ${ }^{2,3,4}$,



${ }^{1}$ Department of Chemical Engineering \& Materials Science, Michigan State University, East Lansing, MI, USA

${ }^{2}$ Institute for Quantitative Health Science and Engineering, Michigan State University, East Lansing, MI, USA

${ }^{3}$ Department of Biomedical Engineering, Michigan State University, East Lansing, MI, USA

${ }^{4}$ Department of Microbiology \& Molecular Genetics, Michigan State University, East Lansing, MI, USA

${ }^{*}$ Corresponding authors.

E-mail addresses: n.ashammakhi@googlemail.com (N. Ashammakhi) and chatzist@msu.edu (X. Chatzistavrou) 
New Bioink

\section{ABSTRACT}

$\overline{\text { Bone tissue loss can occur due to disease, trauma or following surgery, in each case }}$ treatment involving the use of bone grafts or biomaterials is usually required. Recent development of three-dimensional (3D) bioprinting (3DBP) has enabled the printing of customized bone substitutes. Bioinks used for bone 3DBP employ various particulate phases such as ceramic and bioactive glass particles embedded in the bioink creating a composite. When composite bioinks are used for 3DBP based on extrusion, particles are heterogeneously distributed causing damage to cells due to stresses created during flow in the matrix of the composite. Therefore, the objective of this study was to develop cell-friendly osteopromotive bioink mitigating the risk of cell damage due to the flow of particles. Towards this end, we have linked organic and inorganic components, gelatin methacryloyl (GelMA) and Ag-doped bioactive glass (Ag-BaG), to produce a hybrid material, GelMA-Ag-BaG (GAB). The distribution of the elements present in the Ag-BaG in the resulting hybrid GAB structure was examined. Rheological properties of the resulting hydrogel and its printability, as well as the degree of swelling and degradation over time, were also evaluated. GAB was compared to GelMA alone and GelMA-AgBaG nanocomposites. Results showed the superiority of the hybrid GAB bioink in terms of homogenous distribution of the elements in the structure, rheological properties, printability, and degradation profiles. Accordingly, this new bioink represents a major advance for bone 3DBP.

Keywords: Bioactive glass, bone, GelMA, biomaterial, tissue engineering 
New Bioink

\section{INTRODUCTION}

A major clinical challenge in orthopedic and craniomaxillofacial (CMF) surgery is the development of critically sized bone defects typically caused by congenital malformations, trauma, infection, cancer, or surgical resection [1, 2]. Multiple treatment approaches have been implemented to address such defects such as the use of allographs [3], however, the gold standard continues to be the use of autografts [4]. Globally, over two million bone graft procedures are performed annually $[5,6]$ with the commonality of such procedures being second only to blood transfusions [7]. The use of autografts is, however, limited by availability, donor-site morbidity [8], and the challenges of creating the required shape (e.g. CMF applications) [9]. While the use of allografts can address the resource limitations and eliminate concerns regarding donorsite morbidity associated with the use of autografts, the use of allografts presents its unique challenges. For example, the use of devitalized allographs not only employs a high-cost laborious process [7], but also results in limited revascularization [10] significantly increasing the risk of incurring an immunological reaction [11] and infection [12]. Treatment strategies for addressing infected bone often require a second revision surgery that not only prolongs recovery time but also has the potential to lead to more severe ramifications such as permanent loss of function and even amputation [13]. Therefore, there is a critical need to develop effective alternative approaches.

To address this, biomaterials have been used, classically as acellular constructs with a focus on identifying biocompatible and bioinert materials [14]. Success has been demonstrated in addressing the shortcomings of traditional biomaterials-based strategies by tackling said challenges through more biomimetic approaches. The 


\section{New Bioink}

advantages of using cell-seeded polymeric tissue engineering constructs were demonstrated in various studies [15-23]. Unfortunately, many of the available polymers are not cell-friendly due to their synthetic origins. Improvements to the cell-friendly nature of biomaterials have been realized through the use of naturally-derived biomaterials such as decellularized tissue matrices [24] or natural polymers such as collagen [25] and gelatin [26]) either incorporated in conjunction with other biomaterials such as synthetic polymers and growth factors or used alone. The enhanced cellfriendly characteristics were in part due to the presence of the tripeptide arginyl-glycylaspartic acid (RGD) peptide sequences [27] in said naturally derived biomaterials known to be an important factor in cell attachment and function. While acellular biomaterial-based approaches have represented important advances in the biomaterials field, the inclusion of cells appears to be critical to further advancements. Furthermore, the use of cell seeding alone has proven to be an inadequate approach given the subsequent premature failure of implants due to inhomogeneous cell distributions.

Three-dimensional printing (3DP) technologies allowed significant advancements to be made in controlling the geometry of tissue engineering. Combined with imaging and design technologies such as computed tomography (CT), magnetic resonance imaging (MRI), and computer-aided design (CAD) [28], 3DP enabled the development of customized bone substitutes. A crucial innovation in the expansion of 3DP technologies for tissue engineering was the advent of 3D bioprinting (3DBP) allowing cell-laden constructs to be 3D printed leading to advances in the engineering of biomimetic living constructs [29-31]. Three-dimensionally bioprinted patient-specific constructs show potential for improving treatment outcomes and accelerating the time of recovery. This 


\section{New Bioink}

technology is expected to revolutionize the biomaterials and biomedical engineering fields with such innovations starting to enter clinical studies [32, 33].

The creation of a printable bioink is achieved through the incorporation of cells into a liquid matrix that undergoes solidification post-print, forming the desired tissue-like constructs $[34,35]$. Most commonly, 3DBP relies on extrusion-based methods for the printing process [36] however such methods are known to reduce cell viability due to the introduction of shear forces during extrusion. The incorporation of solid elements into bioinks such as glass or ceramic particles that may support bone growth, osteopromotive, leads to increased shear forces during 3DBP leading to additional decreases in cell viability [37].

Typically, bioinks used for 3DBP of bone-like constructs incorporate osteopromotive elements $[38,39]$ such as Ca-based bioceramics $[40,41]$ or silicate-based biomaterials $[42,43]$ in the form of either micro-sized [43] or nano-sized particles [31, 35, 37, 41, 42]. The particles are introduced into the cell-laden polymer matrix through mixing allowing composites to be 3DBP [44]. For example, nanoparticles (NPs) of silicate glasses have been combined with gelatin methacryloyl (GeIMA) to form nanocomposites with the two components held together by ionic interactions [45]. Such nanocomposite bioinks are limited to the degree of homogeneity they can achieve not only by the size of the particles incorporated but also by the degree of agglomeration [37, 46]. This was evidenced by the increase in dead osteoblasts after the extrusion 3DBP of bioactive glass (BaG) containing cell-laden bioinks as a result of the increased shear forces [37] introduced by the $\mathrm{BaG}$ particles [37]. Therefore, alternative strategies to incorporating osteopromotive elements need to be investigated. 


\section{New Bioink}

An innovative approach to overcoming these limitations is to chemically link the osteopromotive component(s) to the polymer matrix. It is expected that the chemical bonding of the osteopromotive component(s) and polymer matrix will result in a stable structure allowing agglomeration of osteopromotive component(s) to be minimized. Furthermore, to achieve the greatest degree of homogenization between the osteopromotive component(s) and the polymer matrix will likely require an in situ synthesis method. Combining these factors should deliver 3DBP bone-like constructs, where the body cannot distinguish the individual components used in the bioink. This would allow for advanced material characteristics to be achieved that could not otherwise have been realized using a composites approach.

$\mathrm{BaG}$ is an attractive osteopromotive component for incorporation into cell-laden bioinks for 3DBP of bone-like constructs given its well-documented improvements in cell viability, osteogenic differentiation, antibacterial properties, and in vivo cell survival, as well as anti-inflammatory, and pro-angiogenic properties [47-53]. The ideal BaGcontaining bioink, therefore, is likely to exhibit the following characteristics: (1) supports the optimal osteogenic response for cells to be induced as a result of the ions provided by the BaG $[50,51]$, (2) increases the stiffness of the 3DBP bone-like constructs [52], possesses the necessary anti-inflammatory characteristics for healing, and (4) allows for the promotion of angiogenesis.

We, therefore, aimed to utilize an innovative approach that combined GelMA with an Ag-doped bioactive glass (GAB) to deliver a novel osteopromotive and antibacterial material. To achieve this, we used a methacryloyl functionalized collagen-derived gelatin (GelMA) as the polymer matrix to both enable crosslinking and enhance cell 


\section{New Bioink}

attachment and function. An Ag-doped bioactive glass (Ag-BaG) was selected for its osteogenic, angiogenic [38, 44, 54], and antibacterial properties [55-57], along with the ability of the Ag-BaG to enhance the strength of the delivered material. The GeIMA and $\mathrm{Ag}-\mathrm{BaG}$ were then chemically linked to produce the hybrid, GAB. Additionally, $\mathrm{Ag}-\mathrm{BaG}$ NPs were mixed with GeIMA to deliver a nanocomposite material, where our developed GAB hybrid material was found to be superior to the synthesized nanocomposite material demonstrating the effectiveness of our innovative approach.

\section{MATERIALS \& METHODS}

\subsection{Material synthesis}

To synthesize GelMA, type A gelatin (300 bloom; Millipore Sigma) was dissolved in dimethyl sulfoxide (DMSO; Millipore sigma) at $50^{\circ} \mathrm{C}$ and stirred at 500 RPM. Methacrylic anhydride (MAA; Millipore Sigma) was added in $1 / 6^{\text {th }}$ increments every 30 minutes to achieve a final MAA:lysine ratio of 2.2 [58]. The resulting solution was then added to toluene at $3 x$ the reaction volume used to induce precipitation of the GelMA [59]. The solution was then decanted after $24 \mathrm{~h}$, washed thrice with distilled water, and then dissolved in distilled water at $50^{\circ} \mathrm{C}$. After dissolution, the solution was placed at $37^{\circ} \mathrm{C}$ for $24 \mathrm{~h}$ to keep the GeIMA dissolved while allowing for any remaining toluene to evaporate. The solution was then frozen and lyophilized before storage.

To synthesize the GAB hybrid material, the lyophilized GelMA was dissolved at $10 \%$ $(w / v)$ in DMSO at $50^{\circ} \mathrm{C}$ and stirred at 500 RPM. A coupling agent, (3Glycidyloxypropyl)trimethoxysilane (GPTMS; Millipore Sigma), was then added to the solution to achieve a hydroxylysine, lysine, arginine to GPTMS ratio of 2.00 and allowed 


\section{New Bioink}

to react for $24 \mathrm{~h}$ to ensure sufficient time for the epoxy ring-opening reaction (Fig. 1). The sol-gel process was used to synthesize the Ag-BaG following previously described methods [56]. $3 \%(\mathrm{w} / \mathrm{w})$ of the $\mathrm{Ag}-\mathrm{BaG}$ sol was added to the GelMA solution in addition to distilled water [56] to ensure sufficient hydrolysis between the GPTMS and Ag-BaG components and allowed to stir for $24 \mathrm{~h}$ to achieve a homogenous distribution between GelMA and $\mathrm{Ag}-\mathrm{BaG}$ at the molecular level (Fig. 1). This solution was then precipitated, washed, and lyophilized as previously described to prevent the materials characteristics from changing during storage (Fig. 1). Prior to further use, lyophilized GAB was dissolved at $10 \%(\mathrm{w} / \mathrm{v})$ in $1 \mathrm{X}$ phosphate buffered saline (PBS) along with the photoinitiator VA-086 at $1.5 \%(\mathrm{w} / \mathrm{v})$ and photopolymerized at $385 \mathrm{~nm}$ for $120 \mathrm{~s}$ producing GAB hydrogels (Fig. 1).

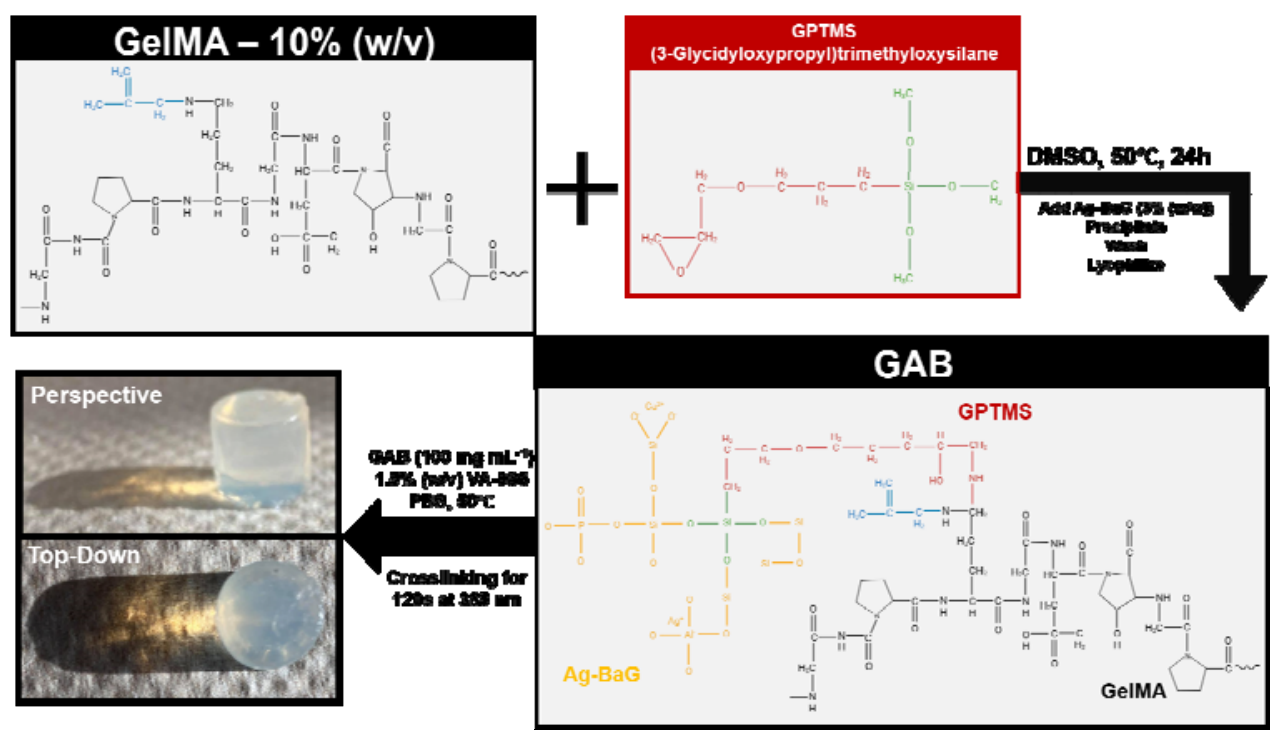

Figure 1. Molecular schematic of the synthesis used to produce GAB along with the process applied to deliver GAB hydrogels.

\subsection{Structural characterization}




\section{New Bioink}

The overall morphological characteristics of the synthesized materials were evaluated using optical microscopy (VHX-600E Digital Microscope) and micro-computerized tomography (Micro-CT; Rigaku Quantum GX).

To study the microscopic morphological features of the synthesized materials, scanning electron microscopy (SEM; Tescan MIRA/JEOL 6610LV) was used. Samples were prepared for SEM examination by first undergoing a graded series of ethanol dehydration (i.e. $2 x-25 \%, 2 x-50 \%, 2 x-75 \%, 2 x-90 \%$, and $3 x 100 \%$ ethanol). The samples were then critically point dried using liquid $\mathrm{CO}_{2}$ in order to preserve the native structure of the materials before being metalized with $\mathrm{Os}_{(\mathrm{g})}$ for $15 \mathrm{~s}$ to prevent a buildup of a negative electrical charge. Sample morphologies were captured using a beam voltage of $5 \mathrm{kV}$. Energy dispersive spectroscopy (EDS; Ametek EDAX Apollo X) was additionally performed to assess elemental homogeneity using a beam voltage of $20 \mathrm{kV}$.

To identify the molecular bonds present within the synthesized samples, Fouriertransformed infrared spectroscopy - attenuated total reflection (FTIR; Jasco FT/IR4600) was applied, where spectra were collected from $4000-400 \mathrm{~cm}^{-1}$. Additionally, spectra relating to the reagents used during the synthesis of GelMA and GAB were collected in order to utilize FTIR for quality control.

Proton nuclear magnetic resonance $\left({ }^{1} \mathrm{H}-\mathrm{NMR}\right)$ was used to determine the methacrylation of gelatin yielded after performing the GeIMA synthesis [60]. The 1D spectra were collected using an Agilent DirectDrive2 $500 \mathrm{MHz}$ NMR spectrometer, where a sample concentration of $50 \mathrm{mg} \mathrm{mL}^{-1}$ in deuterium oxide $\left(\mathrm{D}_{2} \mathrm{O}\right)$ was used for all measurements. The degree of substitution (DS) was calculated using the lysine integral method, where the integral of the lysine methylene signals of the gelatin as-received 


\section{New Bioink}

was compared to the integral of the lysine methylene signals in GelMA, as described in equation 1 [61].

$$
\mathrm{DS}_{\text {Lysine }}=\left(1-\frac{\int \text { Lysine GelMA }}{\int \text { Lysine Gelatin }}\right) * 100
$$

\subsection{Performance characterization}

To assess the swelling behavior of the synthesized samples, lyophilized samples were measured to quantify the mass of the lyophilized samples. The lyophilized samples were hydrated in 1X PBS forming samples $8 \mathrm{~mm}$ in diameter having a thickness of 1.5 $\mathrm{mm}$ and kept in solution for $24 \mathrm{~h}$ at $37^{\circ} \mathrm{C}$. The hydrated samples were removed from the solution and excess PBS was gently blotted from the surface of the samples. The swelling ratio of the samples was calculated using equation 2.

$$
\frac{\left(\mathbf{W}_{\mathrm{t}}-\mathbf{W}_{\mathbf{0}}\right)}{\mathbf{W}_{\mathbf{0}}}
$$

In equation $2, \mathrm{~W}_{\mathrm{t}}$ represents the weight of the swollen sample after $24 \mathrm{~h}$ of immersion in PBS, and $W_{0}$ represents the weight of the lyophilized (dry) sample.

Samples were immersed in $1 \mathrm{X}$ PBS at $37^{\circ} \mathrm{C}$ under shaking at $175 \mathrm{RPM}$ to study their degradation behavior. After $1,3,5,7,14,21$, and $28 \mathrm{~d}$ of immersion, the $\mathrm{pH}$ was measured, and extracts were collected at each time point to elucidate the ion release profile by inductively coupled plasma - optical emission spectrometer (ICP-OES). The samples at each time point were weighted to determine the mass loss profile and determine the time point at which complete degradation is expected.

The viscoelastic behavior of the samples was evaluated using rheological means. To this end, the storage (G') and loss modulus (G") of the samples was 


\section{New Bioink}

evaluated as a function of temperature ranging from ambient conditions to $40^{\circ} \mathrm{C}$. The viscosity of the samples was evaluated at $37^{\circ} \mathrm{C}$ using a shear rate from 0.1 to $100 \mathrm{~s}^{-1}$.

The compressive behavior of the samples was evaluated using fully cross-linked samples. Samples $8 \mathrm{~mm}$ in diameter having a thickness of $1.5 \mathrm{~mm}$ were used and the compression testing was performed using a United SFM electromechanical series universal testing machine with a $20 \mathrm{~N}$ load cell. A constant cross-head speed of $0.5 \mathrm{~mm}$ $\mathrm{s}^{-1}$ was used and all samples were compressed up to $60 \%$ strain. The elastic modulus was determined by the slope of the linear curve in the elastic region.

\subsection{Printability}

The printability of the samples was investigated by varying the pressure (psi), needle gauge, extruder temperature $\left({ }^{\circ} \mathrm{C}\right)$, speed $\left(\mathrm{mm} \mathrm{s}^{-1}\right)$, layer height $(\mathrm{mm})$, cross-linking time (s), and cross-linking intensity (\%) during 3D printing (Allevi 3). Semi-quantification of the printability was performed according to the previously reported procedures [62]. In brief, the circularity of the printed enclosed area was calculated using equation 3.

$$
C=\frac{4 \pi A}{L^{2}}
$$

In equation $3, \mathrm{~L}$ represents the perimeter, $\mathrm{A}$ represents the area, and $\mathrm{C}$ represents the circularity, where a circularity of 1 represents a perfectly circular entity. Given the circularity for squared shapes is equal to $1 / 4 \mathrm{~A}$, the printability parameter $\left(P_{r}\right)$, which is a quantification of similarity of the nature of square-formed prints, was calculated using equation 4.

$$
P_{r}=\frac{L^{2}}{16 A}
$$


New Bioink

The optimal printability is achieved when $\mathrm{P}_{r}=1$ and denotes that the ideal viscosity during the print was achieved. It is important to note, as well, that when $P_{r}>1$, the viscosity during the print was too high, and $\mathrm{P}_{\mathrm{r}}<1$ denoting the viscosity was too low during the print.

\section{RESULTS \& DISCUSSION}

Figure 2a showed the ${ }^{1} \mathrm{H}-$ NMR spectra of the as-received gelatin, the synthesized GelMA, and the GAB. Using equation 1, the degree of substitution for GeIMA and GAB using the lysine integral method was found to be $\sim 100 \%$ providing supporting evidence that the GAB synthesis did not compromise the degree of substitution.

Traditionally, GeIMA has been synthesized in an aqueous environment such as PBS [63] and using methacrylic anhydride (MAA) as the methacrylation agent, leading to a maximum achievable DS of $\sim 80-85 \%$ with large batch-to-batch variations. This was found to be a consequence of using an aqueous environment for the GelMA synthesis as the methacrylic anhydride preferentially hydrolyzes with its aqueous environment to form methacrylic acid, which is non-reactive with gelatin. To overcome this and achieve a DS of $~ 80-85 \%$ required using a $10-32$-fold molar excess of MAA, compared to the lysine groups present in the gelatin [64]. Lengthy dialysis ( $>7 d)$ process was needed to remove the remaining cytotoxic reagents (i.e. MAA). Recently, it has been demonstrated that the use of a carbonate-bicarbonate system tuned to have a pH of 9.0 found success in increasing the DS up to $97 \%$ while dramatically lowering the MAA required to a 2.2 molar excess [64], however, a lengthy dialysis post-processing step was still required. 


\section{New Bioink}

Here, we aimed to remove the adverse effects created by synthesizing GelMA in an aqueous environment through the use of an organic solvent, DMSO. It was hypothesized that the use of DMSO would prevent MAA hydrolysis and allow for $\sim 100 \%$ DS to be achieved during synthesis. Furthermore, it was recently found that the dialysis step could be circumvented when using toluene as a precipitating agent [59]. The small dielectric constant for toluene $(\varepsilon \sim 2.4)$, when exposed to the GelMA solution, increases the attractive forces between the oppositely charged portions of the GeIMA allowing for agglomerations to grow resulting in the precipitation of the GelMA. An additional benefit to using the toluene precipitation method is that the DMSO and MAA are soluble in toluene allowing for their removal without the need for a dialysis process.

To verify the effectiveness of the toluene precipitation method, FTIR spectra (Fig. 2b) of gelatin as-received, GeIMA, and GAB were collected in addition to the reagents used during the synthesis process of GeIMA and GAB. As shown in Figure $\mathbf{2 b}$, minimal spectroscopic changes were observed after the synthesis of GelMA; an indication that the precipitation method used was successful at extracting the GeIMA from the solution. Additionally, the characteristic spectroscopic peaks for MAA and DMSO were not observed in the FTIR spectrum of GelMA (Fig. 2b) providing further evidence that the precipitation method was successful. For the GAB, the FTIR spectrum (Fig. 2b) presented the characteristic amide I, amide II, and amide III groups at $\sim 1650 \mathrm{~cm}^{-1}$, $\sim 1500 \mathrm{~cm}^{-1}$, and $\sim 1450 \mathrm{~cm}^{-1}$ respectively [60] demonstrating the GelMA structure was preserved during the GAB synthesis, as expected. The broad Si-O bending peak 750 $800 \mathrm{~cm}^{-1}[60]$ is characteristic of the $\mathrm{Ag}-\mathrm{BaG}$ indicative that there was successful incorporation and preservation of $\mathrm{Ag}-\mathrm{BaG}$ during precipitation. 
New Bioink

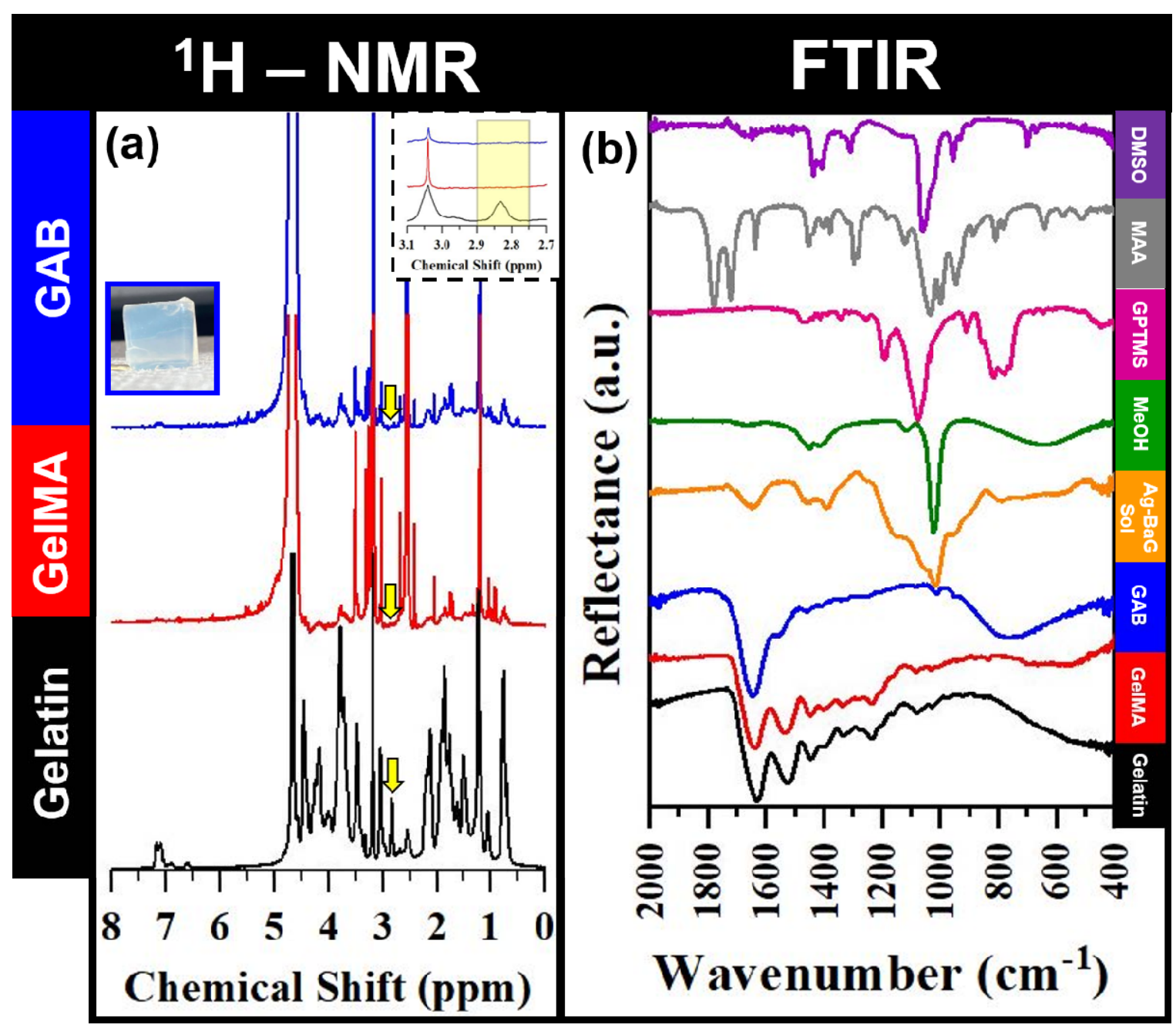

Figure 2. (a) ${ }^{1} \mathrm{H}$ - NMR spectra of gelatin (as-received), GelMA, and GAB. Optical image inserts are additionally included to show the status of the hydrogels postsynthesis. The yellow arrows $(\Longrightarrow)$ denote the lysine methylene signals used to determine the degree of substitution. A zoomed in spectra within this range is additionally shown as an insert for clarity. (b) FTIR spectra of the gelatin (as-received), GeIMA, GAB, the $\mathrm{Ag}-\mathrm{BaG}$ solution used during the $\mathrm{GAB}$ synthesis, methanol $(\mathrm{MeOH})$, the coupling agent (3-Glycidyloxypropyl)trimethoxysilane (GPTMS), methacrylic anhydride (MAA) used for the methacrylation of the as-received gelatin, and dimethyl sulfoxide (DMSO) used as the solvent for the synthesis of GelMA and GAB. 
New Bioink

Given the successful synthesis of GelMA and GAB, when examining the morphology of both on the microscopic scale (Fig. 3a-b, g-h), clear morphological differences were observed. The GAB (Fig. $\mathbf{3 g}-\mathbf{h}$ ) showed thicker filaments of material roughly one micron in thickness with relative uniformity likely a result of the combination of GelMA and Ag-BaG. Interestingly, the combination of the Ag-BaG with GelMA led to a smoother surface morphology of the filaments compared to GelMA alone, where more node-like features were observed. The pore size of the GAB (Fig. 3h) was additionally present on the micron-scale, whereas the pores for GelMA (Fig. 3b) were evident on the sub-micron scale as a result of the sub-micron thick filaments.

Regarding elemental homogeneity, both GelMA (Fig. 3c-f) and GAB (Fig. 3i-q) were found to have all elements homogenously distributed down to the micron level. This provides supporting evidence that the synthesis used to deliver GAB was additionally successful at achieving a high degree of homogeneity. This was critical as this provides supporting evidence that GAB should give a homogenous response when studied in vivo; allowing for the benefits of the GeIMA and $\mathrm{Ag}-\mathrm{BaG}$ to be exhibited simultaneously. 


\section{New Bioink}



Figure 3. SEM images of $(a, b)$ GelMA in addition to the respective EDS X-ray mapping, where (c) $\mathrm{C},(\mathrm{d}) \mathrm{N},(e) \mathrm{O}$, and (f) $\mathrm{Cl}$ were all found to be homogenously distributed down to the micron level. Additionally, SEM images of $(g, h)$ GAB and corresponding EDS X-Ray mapping showing that (i) $\mathrm{C},(\mathrm{j}) \mathrm{N},(\mathrm{k}) \mathrm{O},(\mathrm{I}) \mathrm{Cl},(\mathrm{m}) \mathrm{Si},(\mathrm{n}) \mathrm{Ca}$, (o) $\mathrm{Al}$, (p) Ag, and (q) $\mathrm{Na}$ are all homogeneously distributed down to the micron-level.

Regarding the performance aspects of GeIMA and GAB in addition to the GelMAAg-BaG nanocomposite, the swelling ratio (Fig. 4a), pH evolution (Fig. 4b), and mass loss (Fig. 4c) were studied. It was found that the GAB underwent the least amount of swelling compared to the GeIMA or the nanocomposite, although the difference in the swelling ratio between the nanocomposite and GAB was insignificant. The presence of the $\mathrm{Ag}-\mathrm{BaG}$ in both the nanocomposite and $\mathrm{GAB}$ decreased the ability of the material to swell given the $\mathrm{Ag}-\mathrm{BaG}$ does not experience swelling when exposed to aqueous environments. The swelling ratio (Fig. 4a) for GAB was likely decreased compared to 


\section{New Bioink}

the nanocomposite as a result of the covalent bonding that existed between the $\mathrm{Ag}-\mathrm{BaG}$ and GelMA as opposed to the weaker Van der Waals interactions between Ag-BaG NPs and GelMA.

The $\mathrm{pH}$ evolution, studied over the course of seven days (Fig. 4b), revealed that GAB presented an evolution that modeled, well, the $\mathrm{pH}$ evolution of GelMA. This was likely due to the covalent bonding present in addition to the fine degree of homogeneity achieved during the synthesis process allowing the GeIMA to mediate the ion release from the $\mathrm{Ag}-\mathrm{BaG}$ compared to the nanocomposite. The GelMA-Ag-BaG nanocomposite displayed a large increase in $\mathrm{pH}$ that peaked $\sim 8.8$ before dropping below a $\mathrm{pH}$ of 8 after $5 \mathrm{~d}$ of immersion. Sol-gel-derived bioactive glasses are known to have a burst release of ions [61] at early time points of immersion creating an alkaline environment, as evidenced in Figure $\mathbf{4 b}$. This presents supporting evidence of the benefits that can be achieved when combining the GelMA with the Ag-BaG to create the hybrid material. Additionally, the GAB was found to be the most stable when the mass loss was studied up to seven days compared to the nanocomposite demonstrating further the advantages of covalently bonding the GelMA with the Ag-BaG.

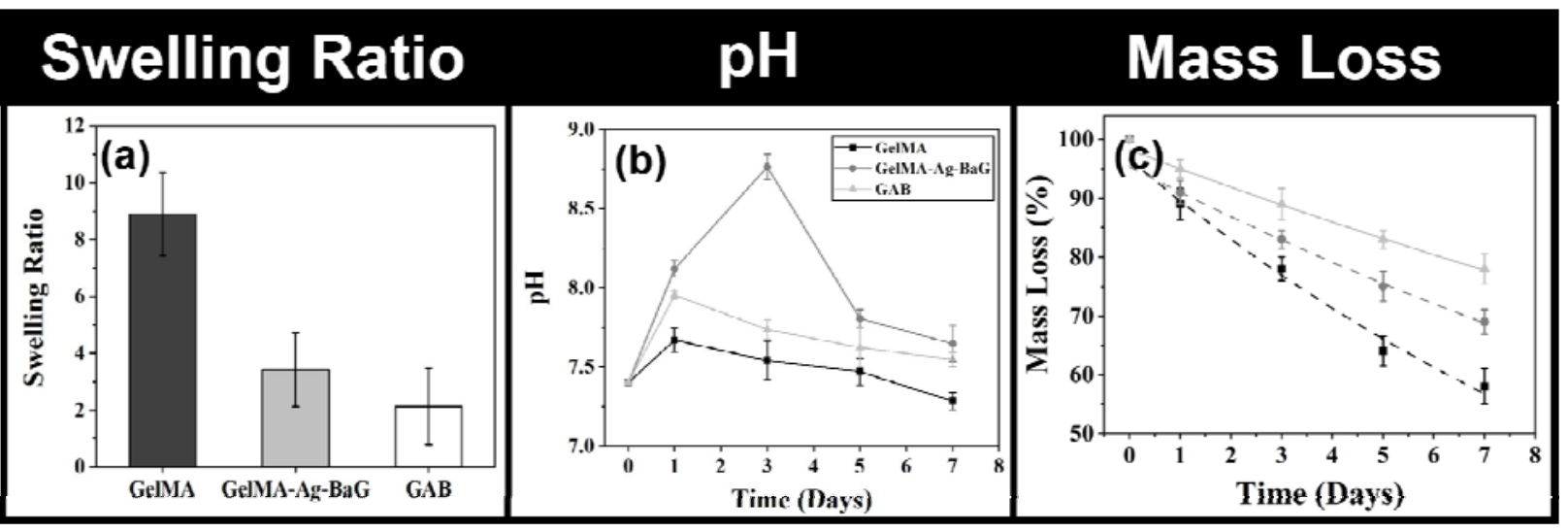

Figure 4. (a) The swelling ratio of GelMA, the GelMA-Ag-BaG nanocomposite, and 
New Bioink

GAB. (b) The pH evolution of GelMA, the GelMA-Ag-BaG nanocomposite, and GAB after 1, 3, 5, and 7d of immersion in PBS in addition to the corresponding (c) mass loss for each time point.

Table 1. Experimental and reported values for the rheological properties of GelMA-containing bioinks.

\begin{tabular}{|c|c|c|c|c|c|c|c|}
\hline Property & \multicolumn{3}{|c|}{$\begin{array}{c}\text { GAB Bioink } \\
10 \%(w / v) \text { GelMA }\end{array}$} & \multicolumn{3}{|c|}{$\begin{array}{l}\text { GelMA Bioink } \\
\text { 10\% (w/v) } \\
\text { Literature }\end{array}$} & $\begin{array}{c}\text { Recommended } \\
\text { Values from } \\
\text { Literature }\end{array}$ \\
\hline Viscosity (Pa.s) & 40,000 & $\sim 1,000$ & $\sim 50$ & $\sim 300$ & $\sim 170$ & $\sim 40$ & $0.03-60,000$ \\
\hline Shear Rate $\left(\mathbf{s}^{-1}\right.$ & 0.1 & 1 & 10 & 0.1 & 1 & 10 & \\
\hline $\begin{array}{c}\text { Storage } \\
\text { Modulus (Pa) }\end{array}$ & \multicolumn{3}{|c|}{$\sim 75,000$} & \multicolumn{3}{|c|}{$\sim 800-1,000$} & $100-1,000$ \\
\hline $\begin{array}{l}\text { Loss Modulus } \\
\qquad(\mathrm{Pa})\end{array}$ & \multicolumn{3}{|c|}{$\sim 20,000$} & \multicolumn{3}{|c|}{$\sim 5-40$} & \\
\hline Shear Thinning & \multicolumn{3}{|c|}{$\checkmark \checkmark \checkmark$} & \multicolumn{3}{|c|}{$\checkmark \checkmark \checkmark$} & $\checkmark \checkmark \checkmark$ \\
\hline
\end{tabular}

To determine whether GAB was a viable material for 3D printing, its rheological performance was studied. Table 1 shows a summary of the viscosity of GAB as a function of shear rate in addition to the storage and loss modulus and compared to values for GelMA obtained from literature along with the recommended values for each materials property for targeting 3D printing applications. 


\section{New Bioink}

For viscoelastic materials such as GelMA and GAB, the storage modulus represents the ability of the material to absorb deformation energy elastically whereas the loss modulus represents the energy dissipated when a force is removed. For GAB, the storage modulus was found to be two orders of magnitude greater than either the GelMA reported in the literature or the recommended values (Table 1). The covalent coupling between GeIMA and Ag-BaG allowed the material to absorb a much greater amount of deformation energy elastically by providing avenues where the strong ionic bonds from the $\mathrm{Ag}-\mathrm{BaG}$ can contribute to the deformation energy storage. The loss modulus for the GAB was expectedly increased compared to GelMA given the presence of the Ag-BaG naturally increases the stiffness as a result of the orders of magnitude higher stiffness for bioactive glasses such as Ag-BaG compared to a softer material, such as GelMA.

The viscosity of the GAB (Fig. $5 \mathbf{b}$ ) was found to decrease as a function of increasing shear rate, an indication of shear-thinning behavior. It is preferential for the GAB to exhibit shear-thinning behavior as shear thinning allows materials to exhibit selfhealing abilities after removal of the shear forces allowing for higher quality constructs to be 3D printed. The linear region present between $\sim 1-10 \mathrm{~s}^{-1}$ (Fig. 5b) was used to determine the flow consistency index $(K)$ and flow behavior index $(n)$ using a power fitting performed following equation 5.

$$
\eta_{\text {app }}=K \dot{\gamma}^{(\mathbf{n}-1)}
$$

From equation $5, \mathrm{GAB}$ was found to have a flow consistency index (K) of $417 \pm 10$ $\left(\mathrm{Pa} \cdot \mathrm{s}^{\mathrm{n}}\right)$ and a flow index behavior of $0.045 \pm 0.007$ with a correlation coefficient $\left(\mathrm{R}^{2}\right)$ of 0.98. Given the low value of $n$ for $G A B$, this is further supporting evidence to suggest 


\section{New Bioink}

that GAB exhibits shear thinning behavior and furthermore behaves as a nonNewtonian fluid.

Figure 5 showed a single-layer mesh 3D printed using GAB, where GAB was successfully printed using a pressure of $15 \mathrm{psi}$, an extruder temperature of $27^{\circ} \mathrm{C}$, a 27 gauge metal tapered-tip, and a printing velocity of $4 \mathrm{~mm} \mathrm{~s}^{-1}$. The ability to 3D print GAB at $15 \mathrm{psi}$ with an extruder temperature only $10^{\circ} \mathrm{C}$ cooler than physiological temperatures should minimize the shear forces and minimize the temperature differential cells would exhibit when incorporated into the material for 3D bioprinting. This presents supporting evidence of the printability of $\mathrm{GAB}$ and demonstrates its potential advantages for $3 \mathrm{D}$ bioprinting compared to other hydrogels such as GelMA. Furthermore, the ability to successfully incorporate $\mathrm{Ag}-\mathrm{BaG}$ into GelMA without requiring the use of NPs is expected to improve cell viability during 3D printing given the introduction of additional shear forces due to the presence of the NPs.

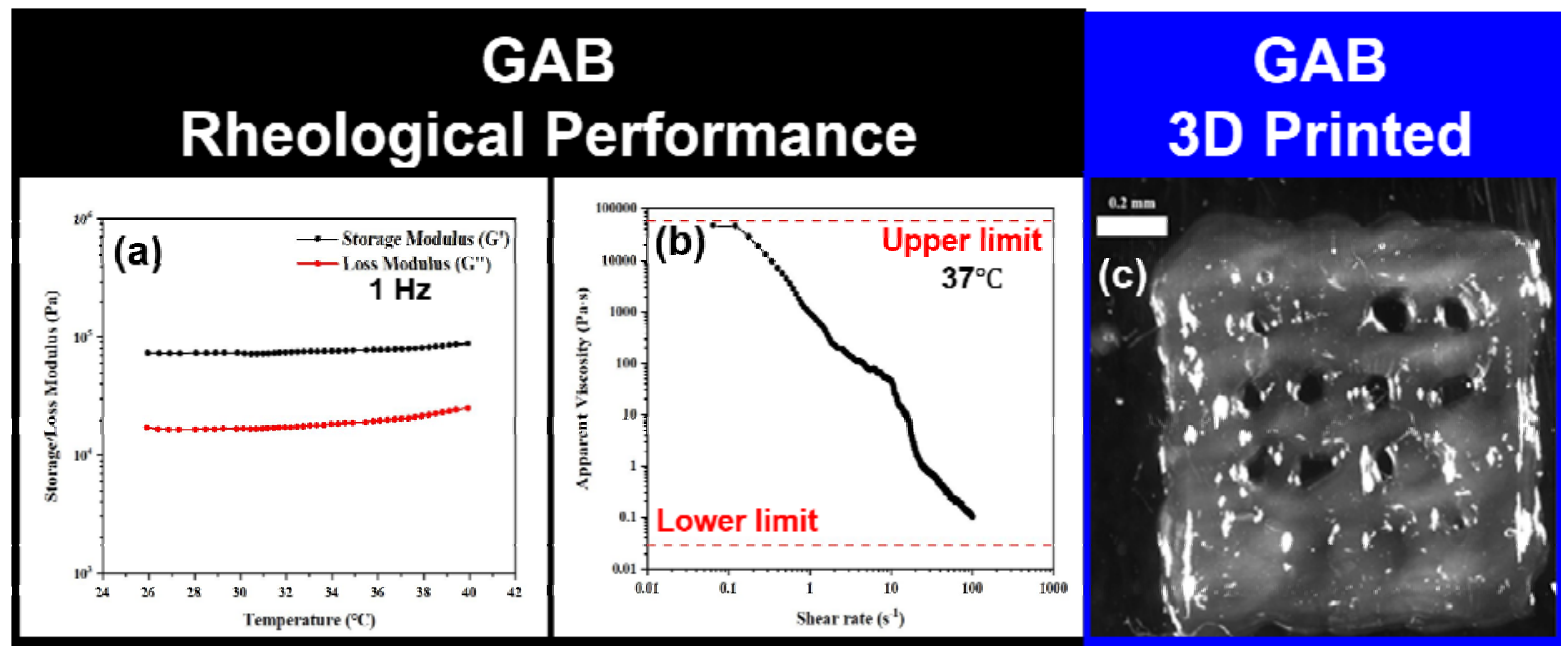

Figure 5. (a) The storage (G') and loss (G') modulus for GAB as a function of temperature ranging from $25^{\circ} \mathrm{C}$ to $40^{\circ} \mathrm{C}$ at $1 \mathrm{~Hz}$ of oscillation. (b) The apparent viscosity of the $\mathrm{GAB}$ as a function of the shear rate showing $\mathrm{GAB}$ exhibits shear thinning 
New Bioink

behavior. (c) A single mesh layer of GAB 3D printed.

\section{CONCLUSIONS}

Following our novel approach, chemically bonded GelMA-Ag-BaG (GAB) were successfully synthesized and cross-linkable enabling 3DP. The rheological evaluation found the GAB exhibited shear thinning behavior, which is a preferential characteristic for printability. The incorporation of the Ag-BaG was found to be homogenous at the molecular level that led the GAB to exhibit the least amount of swelling and the slowest degradation behavior compared to either the GelMA alone or the GelMA-Ag-BaG NP nanocomposites. GAB is expected to be suitable for extrusion-based 3DBP technologies and expected to improve cell viability of the $3 \mathrm{D}$ printed constructs as a result of the improvements in the characteristics of the material of the GAB bioink over a GelMA-Ag-BaG NP nanocomposite bioink.

\section{CONFLICT OF INTEREST}

The authors have no conflict of interest.

\section{REFERENCES}

1. Arealis, G. and V.S. Nikolaou, Bone printing: new frontiers in the treatment of bone defects. Injury, 2015. 46 Suppl 8: p. S20-2.

2. Bara, J.J., et al., A doxycycline inducible, adenoviral bone morphogenetic protein-2 gene delivery system to bone. J Tissue Eng Regen Med, 2018. 12(1): p. e106-e118.

3. Tomford, W.W., Bone Allografts: Past, Present and Future. Cell and Tissue Banking, 2000. 1(2): p. 105-109.

4. Schmidt, A.H., Autologous bone graft: Is it still the gold standard? Injury, 2021. 


\section{New Bioink}

5. Fushimi, H., et al., Recombinant collagen polypeptide as a versatile bone graft biomaterial. Communications Materials, 2020. 1(1): p. 87.

6. Giannoudis, P.V., H. Dinopoulos, and E. Tsiridis, Bone substitutes: An update. Injury, 2005. 36(3, Supplement): p. S20-S27.

7. Campana, V., et al., Bone substitutes in orthopaedic surgery: from basic science to clinical practice. Journal of Materials Science: Materials in Medicine, 2014. 25(10): p. 2445-2461.

8. Betz, R.R., Limitations of Autograft and Allograft: New Synthetic Solutions. Orthopedics, 2002. 25(5): p. S561-S570.

9. Boccardi, E., et al., Characterisation of Bioglass based foams developed via replication of natural marine sponges. Advances in Applied Ceramics, 2015. 114(sup1): p. S56-S62.

10. De Long, W.G., Jr., et al., Bone grafts and bone graft substitutes in orthopaedic trauma surgery. A critical analysis. J Bone Joint Surg Am, 2007. 89(3): p. 649-58.

11. Kurien, T., R.G. Pearson, and B.E. Scammell, Bone graft substitutes currently available in orthopaedic practice: the evidence for their use. Bone Joint J, 2013. 95-b(5): p. 58397.

12. Ng, V.Y., Risk of Disease Transmission With Bone Allograft. Orthopedics, 2012. 35(8): p. 679-681.

13. Thakore, R.V., et al., Surgical site infection in orthopedic trauma: A case-control study evaluating risk factors and cost. J Clin Orthop Trauma, 2015. 6(4): p. 220-6.

14. Ashammakhi, N. and P. Törmälä, From past to present and future is today: from inert to multifunctional biomaterials. J Craniofac Surg, 2004. 15(6): p. 897.

15. Roseti, L., et al., Scaffolds for Bone Tissue Engineering: State of the art and new perspectives. Mater Sci Eng C Mater Biol Appl, 2017. 78: p. 1246-1262.

16. Atala, A., F.K. Kasper, and A.G. Mikos, Engineering complex tissues. Sci Transl Med, 2012. 4(160): p. 160rv12.

17. Bouet, G., et al., In vitro three-dimensional bone tissue models: from cells to controlled and dynamic environment. Tissue Eng Part B Rev, 2015. 21(1): p. 133-56.

18. Henkel, J., et al., Bone Regeneration Based on Tissue Engineering Conceptions - A 21st Century Perspective. Bone Research, 2013. 1(3): p. 216-248.

19. Patricio, T., et al., Fabrication and characterisation of PCL and PCL/PLA scaffolds for tissue engineering. Rapid Prototyping Journal, 2014. 20(2): p. 145-156.

20. Guarino, V., et al., Bio-inspired composite and cell instructive platforms for bone regeneration. International Materials Reviews, 2012. 57(5): p. 256-275.

21. Gonzálvez-García, M., et al., Preclinical Studies of the Biosafety and Efficacy of Human Bone Marrow Mesenchymal Stem Cells Pre-Seeded into B-TCP Scaffolds after Transplantation. Materials, 2018. 11(8): p. 1349.

22. Basu, J., et al., Preclinical biosafety evaluation of cell-based therapies: emerging global paradigms. Toxicol Pathol, 2015. 43(1): p. 115-25.

23. N Ashammakhi, A.G., R Tutar, A Fricker, I Roy, X Chatzistavrou, E Hoque Apu, K-L Nguyen, T Ahsan, I Pountos, E Caterson, Highlights on Advancing Frontiers in Tissue Engineering. Tissue Engineering Part B: Reviews, 2021.

24. Crapo, P.M., T.W. Gilbert, and S.F. Badylak, An overview of tissue and whole organ decellularization processes. Biomaterials, 2011. 32(12): p. 3233-3243.

25. Glowacki, J. and S. Mizuno, Collagen scaffolds for tissue engineering. Biopolymers, 2008. 89(5): p. 338-344. 


\section{New Bioink}

26. Kang, H.-W., Y. Tabata, and Y. Ikada, Fabrication of porous gelatin scaffolds for tissue engineering. Biomaterials, 1999. 20(14): p. 1339-1344.

27. Klotz, B.J., et al., Gelatin-Methacryloyl Hydrogels: Towards Biofabrication-Based Tissue Repair. Trends Biotechnol, 2016. 34(5): p. 394-407.

28. Wang, C., et al., 3D printing of bone tissue engineering scaffolds. Bioact Mater, 2020. 5(1): p. 82-91.

29. Ashammakhi, N., et al., Advancing Frontiers in Bone Bioprinting. Adv Healthc Mater, 2019. 8(7): p. e1801048.

30. Ashammakhi, N., et al., Advancing Frontiers in Bone Bioprinting. Adv. Healthc Mater, 2019: p. e1801048.

31. Potyondy, T., et al., Recent advances in 3D bioprinting of musculoskeletal tissues. Biofabrication, 2021. 13(2): p. 022001.

32. Masaeli, R., et al., Challenges in Three-Dimensional Printing of Bone Substitutes. Tissue Eng Part B Rev, 2019. 25(5): p. 387-397.

33. Tellisi, N., et al., Three Dimensional Printed Bone Implants in the Clinic. J Craniofac Surg, 2018. 29(8): p. 2363-2367.

34. Guvendiren, M., et al., Designing Biomaterials for 3D Printing. ACS Biomaterials Science \& Engineering, 2016. 2(10): p. 1679-1693.

35. Ashammakhi, N., et al., Bioinks and bioprinting technologies to make heterogeneous and biomimetic tissue constructs. Materials Today Bio, 2019. 1: p. 100008.

36. Tavafoghi, M., et al., Multimaterial bioprinting and combination of processing techniques towards the fabrication of biomimetic tissues and organs. Biofabrication, 2021. 13(4).

37. Ashammakhi, N., et al., Advancing Frontiers in Bone Bioprinting. Advanced Healthcare Materials, 2019. 8(7): p. 1801048.

38. Heid, S. and A.R. Boccaccini, Advancing bioinks for 3D bioprinting using reactive fillers: A review. Acta Biomaterialia, 2020. 113: p. 1-22.

39. Ashammakhi, N. and O. Kaarela, Three-Dimensional Bioprinting Can Help Bone. J Craniofac Surg, 2018. 29(1): p. 9-11.

40. Midha, S., S. Murab, and S. Ghosh, Osteogenic signaling on silk-based matrices. Biomaterials, 2016. 97: p. 133-53.

41. Demirtaş, T.T., G. Irmak, and M. Gümüşderelioğlu, A bioprintable form of chitosan hydrogel for bone tissue engineering. Biofabrication, 2017. 9(3): p. 035003.

42. Byambaa, B., et al., Bioprinted Osteogenic and Vasculogenic Patterns for Engineering 3D Bone Tissue. Advanced Healthcare Materials, 2017. 6(16): p. 1700015.

43. Wang, X., et al., Effect of Bioglass on Growth and Biomineralization of SaOS-2 Cells in Hydrogel after 3D Cell Bioprinting. PLOS ONE, 2014. 9(11): p. e112497.

44. Murphy, C., et al., 3D bioprinting of stem cells and polymer/bioactive glass composite scaffolds for bone tissue engineering. 2017, 2017. 3(1): p. 11.

45. Tavares, M.T., et al., GelMA/bioactive silica nanocomposite bioinks for stem cell osteogenic differentiation. Biofabrication, 2021. 13(3): p. 035012.

46. Hassan, M., et al., 3D printing of biopolymer nanocomposites for tissue engineering: Nanomaterials, processing and structure-function relation. European Polymer Journal, 2019. 121: p. 109340. 


\section{New Bioink}

47. Haro Durand, L., et al., In vitro human umbilical vein endothelial cells response to ionic dissolution products from lithium-containing 45S5 bioactive glass. Materials, 2017. 10(7): p. 740.

48. Gerhardt, L.-C., et al., The pro-angiogenic properties of multi-functional bioactive glass composite scaffolds. Biomaterials, 2011. 32(17): p. 4096-4108.

49. Chandrasekar, R.S., et al., Evaluation of antimicrobial properties of bioactive glass used in regenerative periodontal therapy. Journal of Indian Society of Periodontology, 2015. 19(5): p. 516.

50. Khazaei, M., et al., Stem cells in dentistry, sources, and applications. Dental Hypotheses, 2016. 7(2): p. 42.

51. Nguyen, L.H., et al., Vascularized bone tissue engineering: approaches for potential improvement. Tissue Engineering Part B: Reviews, 2012. 18(5): p. 363-382.

52. Tavakoli, E., et al., Preparation, characterization and mechanical assessment of poly (lactide-co-glycolide)/hyaluronic acid/fibrin/bioactive glass nano-composite scaffolds for cartilage tissue engineering applications. Procedia Materials Science, 2015. 11: p. 124130.

53. Santos-Ruiz, L., et al., Potential use of craniosynostotic osteoprogenitors and bioactive scaffolds for bone engineering. J Tissue Eng Regen Med, 2007. 1(3): p. 199-210.

54. Vuornos, K., et al., Bioactive glass ions induce efficient osteogenic differentiation of human adipose stem cells encapsulated in gellan gum and collagen type I hydrogels. Materials Science and Engineering: C, 2019. 99: p. 905-918.

55. Pajares-Chamorro, N., et al., Resurrection of antibiotics that methicillin-resistant Staphylococcus aureus resists by silver-doped bioactive glass-ceramic microparticles. Acta Biomater, 2019. 96: p. 537-546.

56. Pajares-Chamorro, N. and X. Chatzistavrou, Bioactive Glass Nanoparticles for Tissue Regeneration. ACS Omega, 2020. 5(22): p. 12716-12726.

57. Pajares-Chamorro, N., et al., Silver-doped bioactive glass particles for in vivo bone tissue regeneration and enhanced methicillin-resistant Staphylococcus aureus (MRSA) inhibition. Mater Sci Eng C Mater Biol Appl, 2021. 120: p. 111693.

58. Lee, B.H., et al., Efficient and controllable synthesis of highly substituted gelatin methacrylamide for mechanically stiff hydrogels. RSC Advances, 2015. 5(128): p. 106094-106097.

59. Mohamed, M.G.A., High-efficiency biofabrication of cell-laden gelatin methacryloyl hydrogels, in Mechanical Engineering. 2020, The University of British Columbia: British Columbia, Canada. p. 126.

60. Claaßen, C., et al., Quantification of Substitution of Gelatin Methacryloyl: Best Practice and Current Pitfalls. Biomacromolecules, 2018. 19(1): p. 42-52.

61. Hoch, E., et al., Stiff gelatin hydrogels can be photo-chemically synthesized from low viscous gelatin solutions using molecularly functionalized gelatin with a high degree of methacrylation. Journal of Materials Science: Materials in Medicine, 2012. 23(11): p. 2607-2617.

62. Ouyang, L., et al., Effect of bioink properties on printability and cell viability for 3D bioplotting of embryonic stem cells. Biofabrication, 2016. 8(3): p. 035020.

63. Van Den Bulcke, A.I., et al., Structural and Rheological Properties of Methacrylamide Modified Gelatin Hydrogels. Biomacromolecules, 2000. 1(1): p. 31-38. 


\section{New Bioink}

64. Shirahama, H., et al., Precise Tuning of Facile One-Pot Gelatin Methacryloyl (GelMA) Synthesis. Scientific Reports, 2016. 6(1): p. 31036.

60. Farmer, V.C. The Infrared Spectra of Minerals, Mineralogical Society of Great Britain and Ireland, London, 1974. https://doi.org/10.1180/mono-4. 9780903056052.

61. Ciraldo, Francesca E., et al. "Tackling bioactive glass excessive in vitro bioreactivity: Preconditioning approaches for cell culture tests." Acta biomaterialia 75 (2018): 3-10. 